\title{
THE MAXIMUM DISTRIBUTION OF A GAUSSIAN STOCHASTIC PROCESS INDEXED BY A LOCAL FIELD
}

\author{
STEVEN N. EVANS \\ (Received 24 November 1986; revised 3 July 1987)
}

Communicated by T. C. Brown

\begin{abstract}
We consider continuous Gaussian stochastic process indexed by a compact subset of a vector space over a local field. Under suitable conditions we obtain an asymptotic expression for the probability that such a process will exceed a high level. An important component in the proof of these results is a theorem of independent interest concerning the amount of 'time' which the process spends at high levels.
\end{abstract}

1980 Mathematics subject classification (Amer. Math. Soc.) (1985 Revision): 60 G 15, 60 G 10, 26 E 30.

The sample path properties of Gaussian stochastic processes indexed by a subset of a finite dimensional vector space over a local field (that is, a non-discrete, locally compact, totally disconnected topological field) have been studied by the author in Evans $(1986,1988)$. A number of the results in these two papers indicate that such processes can exhibit behaviour which has no analogue for processes indexed by a Euclidean space. Other results do have Euclidean counterparts but the proofs are sometimes quite dissimilar and often considerably simpler in the local field case. Also, as well as being of intrinsic interest, these processes can provide a useful tool for constructing interesting examples of sets and functions in the analysis of local fields.

Let $X$ be a continuous Gaussian process by a compact subset of a finite dimensional vector space over a local field. An important input into several of the

(C) 1989 Australian Mathematical Society 0263-6115/89 \$A2.00+0.00 
results in Evans $(1986,1988)$ was a fairly crude upper bound on a probability that $X$ will exceed a high level (see Corollary (9-2) of Evans (1988) which is restated as Lemma (2-7) below). In the present paper we show that it is sometimes possible to say considerably more about the tail of the maximum of $X$. In Theorem (4-1) we show that if $X$ is stationary then under an approximate scaling condition on the covariance kernel of $X$ we may find explicit sequences $\left.\{u(n)\}_{n=1}^{\infty} \subset\right] 0, \infty[$ and $\{p(n)\}_{n=1}^{\infty}$ such that $u(n) \rightarrow \infty$ as $n \rightarrow \infty$ and

$$
\lim _{n \rightarrow \infty} P\left(\max _{t} X(t)>u(n)\right) / p(n)=1 .
$$

In Corollary (4-7) we replace the condition that $X$ is stationary by the much weaker condition that $E\left(X(t)^{2}\right)$ is constant and show that under appropriate assumptions on the growth of the incremental variance function of $X$ we may find explicit sequences $\left.\{u(n)\}_{n=1}^{\infty} \subset\right] 0, \infty\left[\right.$ and $\{p(n)\}_{n=1}^{\infty}$ such that $u(n) \rightarrow \infty$ and $n \rightarrow \infty$ and

$$
\begin{aligned}
0 & <\liminf _{n \rightarrow \infty} P\left(\max _{t} X(t)>u(n)\right) / p(n) \\
& \leq \limsup _{n \rightarrow \infty} P\left(\max _{t} X(t)>u(n)\right) / p(n)<\infty .
\end{aligned}
$$

The techniques which we use to obtain Theorem (4-1) are similar to those used in Berman (1982) to obtain corresponding results for processes indexed by subsets of ] $-\infty, \infty$ [. In particular, we rely on detailed asymptotics for the amount of 'time' that the process sojourns above high levels. These are obtained in Section 3 using methods from Berman (1985).

We remark that Theorem (4-1) complements the results of Berman as well as those obtained by other authors in the Euclidean case (see, for example, Pickands (1969a,b) or Belyaev and Piterbarg (1972)) in that they indicate how it is the concordance of the covariance structure of the process with the translation and dilation structure of the index space (that is, stationarity and approximate scaling) which underlies the proofs in both settings.

On the other hand, Corollary (4-7) is of a more novel nature in that there appears to be a dearth in the Euclidean literature of general results giving nontrivial asymptotic lower bounds on the probability that a non-stationary process will exceed a high level.

\section{Local fields}

This section is more or less a summary of results to be found in Taibleson (1975) and we refer the reader to this latter account for the relevant proofs and references. 
Let $K$ be a topological field, that is, $K$ is a field such that $K^{+}$and $K^{*}$ are topological groups (where $K^{+}$and $K^{*}$ are the additive and multiplicative groups of $K$ ). If $K$ is locally compact, non-discrete and connected then $K$ is either $\mathbf{R}$ or C. If $K$ is locally compact, non-discrete and disconnected then $K$ is totally disconnected and we say that $K$ is a local field.

For the rest of this section let $K$ be a fixed local field. There is a distinguished real-valued mapping on $K$ which we denote by $x \mapsto|x|$ and call the valuation map. This map takes values in the set $\left\{q^{k}: k \in \mathbf{Z}\right\} \cup\{0\}$, where $q=p^{c}$ for some prime $p$ and positive integer $c$, and has the following properties:

$$
\begin{gathered}
|x|=0 \Leftrightarrow x=0 ; \\
|x y|=|x||y| ; \\
|x+y| \leq \max [|x|,|y|] .
\end{gathered}
$$

The last property is called the ultrametric inequality and it implies that if $|x| \neq$ $|y|$ then $|x+y|=\max [|x|,|y|]$. The mapping $(x, y) \mapsto|x-y|$ on $K \times K$ is a metric on $K$ which gives the topology of $K$.

The set $D=\{x \in K:|x| \leq 1\}$, which we call the ring of integers in $K$, is the unique maximal compact subring of $K$. There exists $\rho \in D$ with $|\rho|=q^{-1}$. We set

$$
B=\{x \in K:|x|<1\}=\left\{x \in K:|x| \leq q^{-1}\right\}=\rho D,
$$

and

$$
B^{k}=\left\{x \in K:|x| \leq q^{-k}\right\}=\rho^{k} D, \quad k \in \mathbf{Z} .
$$

Each $B^{k}$ is compact and open and a subgroup of $K^{+}$. If $k>k^{\prime}$ then $B^{k}$ is an additive subgroup of $B^{k^{\prime}}$ with $\operatorname{card}\left(B^{k^{\prime}} / B^{k}\right)=q^{k-k^{\prime}}$.

Let $K^{N}$ be the canonical $N$-dimensional vector space over $K$. For $x=$ $\left(x^{1}, \ldots, x^{N}\right) \in K^{N}$ the mapping $x \mapsto|x|=\max _{i}\left|x^{i}\right|$ satisfies:

$$
\begin{gathered}
|x|=0 \Leftrightarrow x=0 ; \\
|\alpha x|=|\alpha||x|, \quad \alpha \in K \\
|x+y| \leq \max [|x|,|y|] .
\end{gathered}
$$

If $|x| \neq|y|$ then $|x+y|=\max [|x|,|y|]$. The mapping $(x, y) \mapsto|x-y|$ on $K^{N} \times K^{N}$ is a metric which gives the product topology of $K^{N}$.

We have $D^{N}=\left\{x \in K^{N}:|x| \leq 1\right\}$ and $\left(B^{k}\right)^{N}=\left\{x \in K^{N}:|x| \leq q^{-k}=\right.$ $\rho^{k} D^{N}, k \in$ Z. If $k>k^{\prime}$ then $\left(B^{\bar{k}}\right)^{N}$ is an additive subgroup of $\left(B^{k^{\prime}}\right)^{N}$ with $\operatorname{card}\left(\left(B^{k^{\prime}}\right)^{N} /\left(B^{k}\right)^{N}\right)=q^{\left(k-k^{\prime}\right) N}$.

There is a distinguished Borel measure $d x$ on $K^{N}$ for which we denote $\int_{A} d x$ by $|A|$. This measure has the properties:

$$
\begin{aligned}
|x+A| & =|A|, \quad x \in K^{N} ; \\
|\alpha A| & =|\alpha|^{N}|A|, \quad \alpha \in K ; \\
\left|D^{N}\right| & =1 .
\end{aligned}
$$


The second of these properties is a special case of the result that $|\Lambda A|=$ $|\operatorname{det} \Lambda||A|$, where $\Lambda$ is a $N \times N$-matrix over $K$ and the determinant is calculated exactly as in the case when the ground field is $\mathbf{R}$ or $\mathbf{C}$.

\section{Preliminary results for Gaussian processes}

For the remainder of this paper we will work with the following class of processes.

DEFINITION. Let $(\Omega, \mathcal{F}, P)$ be a complete probability space. We say a stochastic process $\left\{X(t): t \in D^{N}\right\}$ is of class $\mathcal{G}$ (or, simply, $X \in \mathcal{G}$ ) if

(i) $X$ is real-valued Gaussian;

(ii) $X$ is centred, that is, $E X(t)=0, t \in D^{N}$;

(iii) $X$ has a continuous covariance kernel.

For ease of reference, we include some results that we will require later. The first of these is a consequence of Proposition (3-2) in Evans (1988) and ensures that we have a plentiful supply of concrete examples of stationary processes.

Proposition (2-1). Suppose that $g:\left\{q^{-k}\right\}_{k \in N} \cup\{0\} \rightarrow[0, \infty[$ is nonincreasing and continuous at 0 . Then there exists $X \in \mathcal{G}$ with stationary covariance kernel

$$
E X(s) X(t)=g(|s-t|), \quad s, t \in D^{N} .
$$

We will be primarily concerned with continuous processes. For the sake of completeness we include the following sufficient condition for sample path continuity which is just a particular case of a result due to Dudley (see for example, Theorem IV-5-2 of Jain and Marcus (1978)).

DEFINITION. Let $T$ be a pseudo-metric space with pseudo-metric $d$. For $\varepsilon>0$ let $\nu(\varepsilon)$ denote the minimum number of balls of radius at most $\varepsilon$ that cover $T$. The function $H(\varepsilon)=\log \nu(\varepsilon)$ is called the metric entropy of $T$ with respect to $d$.

THEOREM (2-2). Consider $X \in \mathcal{G}$. Let $H$ denote the metric entropy of $D^{N}$ with respect to the pseudo-metric $\sigma$, where

$$
\sigma^{2}(s, t)=E(X(s)-X(t))^{2}
$$

is the incremental variance function of $X$. The condition

$$
\int_{0}^{1} H(u)^{1 / 2} d u<\infty
$$


implies that there exists a version $\left\{\tilde{X}(t): t \in D^{N}\right\}$ of $X$ with continuous sample paths such that for every $\omega \in \Omega$ there exists a $\delta(\omega)>0$ for which

$$
\sigma(s, t) \leq \delta(\omega) \Rightarrow|\tilde{X}(s, \omega)-\tilde{X}(t, \omega)| \leq 147 \int_{0}^{\sigma(s, t)} H(u)^{1 / 2} d u
$$

A related result to Theorem (2-2) is the following theorem which is a special case of Theorem IV-5-5 in Jain and Marcus (1978).

THEOREM (2-3). Consider a continuous process $X \in \mathcal{G}$. In the notation of Theorem $(2-2)$ we have for $0<\delta \leq 1 / 2$ that

$$
E\left(\sup _{\sigma(s, t)<\delta}|X(s)-X(t)|\right) \leq \kappa\left(\int_{0}^{\delta} H(u)^{1 / 2} d u+\delta\left(\log \log \delta^{-1}\right)^{1 / 2}\right),
$$

where $\kappa$ is a fixed constant which does not depend on $X$.

In order to apply Theorems (2-2) and (2-3) it is helpful to have some simple means of estimating the integrals that appear. The following is an analogue of Lemma IV-5-3 in Jain and Marcus (1978) (see Lemma (7-2) in Evans (1988)).

LEMMA (2-4). Let $\sigma$ and $H$ be as in the statement of Theorem (2-2). Suppose that

$$
\sigma(s, t) \leq s(|s-t|), \quad s, t \in D^{N}
$$

for some non-decreasing function $\zeta:[0,1] \rightarrow \mathbf{R}$. Then for $0<\delta<1,|s-t|<\delta$,

$$
\int_{0}^{\sigma(s, t)} H(u)^{1 / 2} d u \leq N^{1 / 2}\left[\varsigma(\delta)(\log q / \delta)^{1 / 2}+\int_{0}^{\delta} \varsigma(u) u^{-1}\left(\log u^{-1}\right)^{-1 / 2} d u\right]
$$

EXAMPLE. Suppose that $\alpha, \beta>0$ are given and $k \in \mathbf{N}$ is such that $q^{k \alpha}>\beta$. From Proposition (2-1), Lemma (2-4) and Theorem (2-2) it is easy to see that there exists a continuous process $X(\cdot ; \alpha, \beta, k) \in \mathcal{G}$ with covariance kernel

$$
E X(s ; \alpha, \beta, k) X(t ; \alpha, \beta, k)= \begin{cases}1-\beta|s-t|^{\alpha}, & \text { if }|s-t| \leq q^{-k} \\ 0, & \text { otherwise }\end{cases}
$$

We will also need a readily checkable criterion for the tightness of a family of Gaussian measures on $C\left(D^{N}, \mathbf{R}\right)$. The requisite result, Corollary (2-6), is obtained by using the estimates of Lemma (2-4) and Theorem (2-3) to verify the conditions of the following general tightness lemma. 
LEMMA (2-5). Let $\left\{P_{n}\right\}_{n=1}^{\infty}$ be a sequence of probability measures on $C(T, \mathbf{R})$, where $(T, d)$ is a compact metric space. Assume that

(i) For each $\eta>0$ there exists $a>0$ such that for all $n$

$$
\sup _{t \in T} P_{n}(x \in C(T, \mathbf{R}):|x(t)|>a) \leq \eta ;
$$

(ii) For each $\varepsilon>0$ and $\eta>0$ there exists $\delta>0$ such that for all $n$

$$
P_{n}\left(x \in C(T, \mathbf{R}): \sup _{s, t \in T, d(s, t) \leq \delta}|x(s)-x(t)|>\varepsilon\right) \leq \eta .
$$

Then the sequence $\left\{P_{n}\right\}_{n=1}^{\infty}$ is tight.

PROOF. Suppose that $\eta>0$ is given. Using (ii) we may find $\delta>0$ such that

$$
P_{n}\left(x: \sup _{d(s, t) \leq \delta}|x(s)-x(t)| \geq 1\right) \leq \eta / 2 .
$$

Let $\left\{t_{1}, \ldots, t_{m}\right\}$ be a $\delta$-net on $T$. Using (i) we may find $a>0$ such that

$$
\sup _{1 \leq j \leq m} P_{n}\left(x:\left|x\left(t_{j}\right)\right|>a\right) \geq \eta / 2 m
$$

for all $n$ and hence $P_{n}\left(x: \sup _{1 \leq j \leq m}\left|x\left(t_{j}\right)\right|>a\right) \leq \eta / 2$ for all $n$. Thus we have $P_{n}\left(x: \sup _{t \in T}|x(t)|>a+1\right) \leq \eta$. In the presence of (ii) we therefore see that (i) is equivalent to the condition:

$\left(\mathrm{i}^{\prime}\right)$ For each $\eta>0$ there exists $a>0$ such that for all $n$

$$
P_{n}\left(x: \sup _{t \in T}|x(t)|>a\right) \leq \eta .
$$

We can now follow the proof of Theorem 8-2 in Billingsley (1968). Let $\eta>0$ be given. Using ( $\left.\mathrm{i}^{\prime}\right)$ choose $a>0$ so that if

$$
B=\left\{x: \sup _{t \in T}|x(t)| \leq a\right\}
$$

then $P_{n}(B)>1-\eta / 2$ for all $n$. Using (ii) choose $\delta(k)>0$ so that if

$$
B_{k}=\left\{x: \sup _{d(s, t) \leq \delta(k)}|x(s)-x(t)| \leq 2^{-k}\right\}
$$

then $P_{n}\left(B_{k}\right)>1-\eta / 2^{k+1}$. Let $F$ denote the closure of $B \cap \bigcap_{k=1}^{\infty} B_{k}$ in $C(T, \mathbf{R})$, then $P_{n}(F)>1-\eta$ for all $n$ and by the Arzelà-Ascoli Theorem $F$ is compact. Hence the sequence $\left\{P_{n}\right\}_{n=1}^{\infty}$ is tight. 
COROLLARY (2-6). Let $\left\{X_{n}\right\}_{n=1}^{\infty}$ be a sequence of continuous class $\mathcal{G}$ processes. Assume that

(i) $\sup _{n} \sup _{t \in D^{N}} E X_{n}(t)^{2}<\infty$;

(ii) There exists a non-decreasing function $\zeta:[0,1] \rightarrow \mathbf{R}$ such that

$$
\left(E\left(X_{n}(s)-X_{n}(t)\right)^{2}\right)^{1 / 2} \leq \varsigma(|s-t|), \quad s, t \in D^{N}, \text { all } n,
$$

and

$$
\int_{0}^{\varepsilon} \varsigma(u) u^{-1}\left(\log u^{-1}\right)^{-1 / 2} d u<\infty
$$

for some $\varepsilon>0$.

Then, letting $P_{n}$ denote the image measure induced by $X_{n}$ on $C\left(D^{N}, \mathbf{R}\right)$, we have that the sequence $\left\{P_{n}\right\}_{n=1}^{\infty}$ is tight.

ProOF. Condition (i) of Lemma (2-5) follows easily from (i) and Chebyshev's inequality. Condition (ii) of Lemma (2-5) follows immediately from Lemma (2-4), Theorem (2-3) and Markov's inequality.

Finally, we will require a general upper bound on the tail of the maximum of a continuous process $X \in \mathcal{G}$ (see Corollary (9-2) of Evans (1988)).

LEMMA (2-7). Let $X \in \mathcal{G}$ be a continuous process. Suppose that

$$
\left(E(X(s)-X(t))^{2}\right)^{1 / 2} \leq \xi(|s-t|)
$$

for some continuous, nondecreasing function $\xi:\left\{q^{-k}\right\}_{k \in N} \cup\{0\} \rightarrow \mathbf{R}$. Set

$$
\alpha=\max _{t \in D N}\left(E X(t)^{2}\right)^{1 / 2} .
$$

Then for $a>(2(2 N+1))^{1 / 2}$ and $m \in\{1,2, \ldots\}$ we have

$$
\begin{aligned}
P\left[\max _{t \in D^{N}}|X(t)|\right. & \left.>a\left(\alpha+\left(1+(2 m \log q)^{-1}\right)^{1 / 2} \sum_{r=1}^{\infty}(\log n[m, r])^{1 / 2} \xi\left(n[m, r]^{-1}\right)\right)\right] \\
& \leq 2\left(q^{2 N m}+\left(q^{m}-1\right)^{-1}\right) \exp \left(-a^{2} / 2\right),
\end{aligned}
$$

where $n[m, r]=q^{m 2^{r}}$.

\section{A sojourn limit theorem}

Suppose that $X \in \mathcal{G}$ is a jointly measurable process. For a sequence $\{A(n)\}_{n=1}^{\infty}$ of Borel subsets of $\mathbf{R}$ we may define a corresponding sequence of sojourn times by

$$
L(n)=\left|\left\{t \in D^{N}: X(t) \in A(n)\right\}\right|, \quad n=1,2, \ldots
$$


We wish to show that under suitable conditions there exists a sequence $\{c(n)\}_{n=1}^{\infty}$ of constants and a non-increasing function $F:] 0, \infty[\rightarrow \mathbf{R}$ such that

$$
\lim _{n \rightarrow \infty} \frac{P(c(n) L(n)>x)}{E(c(n) L(n))}=F(x)
$$

at each continuity point $x$ of $F$

Results of this kind for processes indexed by subsets of $\mathbf{R}^{N}$ have been developed over a long period of time in the researches of Berman (see, for example, Berman (1985) for a brief history and bibliography). Our approach follows that to be found in Berman (1985).

LEMMA (3-1). If there exists a non-decreasing function $G:] 0, \infty[\rightarrow \mathbf{R}$ such that

$$
\lim _{n \rightarrow \infty} \frac{\int_{0}^{x} y d P(c(n) L(n) \leq y)}{E(c(n) L(n))}=G(x)
$$

at each continuity point $x$ of $G$ then

$$
\lim _{n \rightarrow \infty} \frac{P(c(n) L(n)>x)}{E(c(n) L(n))}=F(x)
$$

at each continuity point $x$ of $F$, where $F:] 0, \infty[\rightarrow \mathbf{R}$ is given by

$$
F(x)=\int_{x}^{\infty} y^{-1} d G(y)
$$

Proof. See Lemma 2-2 of Berman (1985).

From now on in this section we assume that our process $X$ is stationary and the sequence $\{A(n)\}$ is such that $P(X(0) \in A(n))>0$ for each $n$ and $\lim _{n \rightarrow \infty} P(X(0) \in A(n))=0$.

NOTATION. For $r>0$ set

$$
L(n ; r)=\left|\left\{t \in D^{N},|t| \leq r: X(t) \in A(n)\right\}\right| .
$$

LEMMA (3-2). Suppose that $\{v(n)\}_{n=1}^{\infty} \subset D \backslash\{0\}$ is such that

$$
\lim _{r \rightarrow \infty} \limsup _{n \rightarrow \infty}|v(n)|^{-N} \int_{\{|t|>r|v(n)|\}} P(X(t) \in A(n) \mid X(0) \in A(n)) d t=0 .
$$

Set

$$
a(x ; n)=\frac{\int_{[0, x]} y d P\left(|v(n)|^{-N} L(n) \leq y\right)}{E\left(|v(n)|^{-N} L(n)\right)}
$$

and

$$
b(x ; n ; r)=P\left(|v(n)|^{-N} L(n ; r|v(n)|) \leq x \mid X(0) \in A(n)\right) .
$$


Assume that there exists a non-decreasing function $B:] 0, \infty[\rightarrow]-\infty, \infty[$ such that $\lim _{r \rightarrow \infty} \lim _{n \rightarrow \infty} b(x ; n ; r)=B(x)$ whenever $x$ is a continuity point of $B$. Then if $z$ is a continuity point of $B$ we have that $\lim _{n \rightarrow \infty} a(z ; n)=B(z)$.

Proof (see Lemma 6-1 in Berman (1985)). From Fubini's Theorem and stationarity we have

$$
\begin{array}{rl}
\int_{0}^{x} y & d P\left(|v(n)|^{-N} L(n) \leq y\right)=E\left(|v(n)|^{-N} L(n) ;|v(n)|^{-N_{d}} L(n) \in[0, x]\right) \\
= & |v(n)|^{-N} E\left(\int_{D^{N}} I_{A(n)}(X(t)) I_{[0, x]}\left(|v(n)|^{-N} L(n)\right) d t\right) \\
& =|v(n)|^{-N} E\left(\int_{D^{N}} I_{A(n)}(X(t)) I_{[0, x]}\left(|v(n)|^{-N} \int_{D^{N}} I_{A(n)}(X(s+t)) d s\right) d t\right) \\
& =|v(n)|^{-N} P\left(|v(n)|^{-N} L(n) \leq x, X(0) \in A(n)\right) .
\end{array}
$$

As

$$
E\left(|v(n)|^{-N} L(n)\right)=|v(n)|^{-N} P(X(0) \in A(n))
$$

we therefore have that

$$
a(x ; n)=P\left(|v(n)|^{-N} L(n) \leq x \mid X(0) \in A(n)\right) ;
$$

and, since $L(n ; r|v(n)|) \leq L(n)$, it is clear that

$$
\limsup _{n \rightarrow \infty} a(x ; n) \leq \lim _{r \rightarrow \infty} \limsup _{n \rightarrow \infty} b(x ; n ; r) .
$$

Conversely, observe that if $\varepsilon \in] 0,1[$ then

$$
\begin{aligned}
& P\left(|v(n)|^{-N} L(n ; r|v(n)|) \leq x(1-\varepsilon) \mid X(0) \in A(n)\right) \\
& \quad \leq\left(|v(n)|^{-N} L(n) \leq x \mid X(0) \in A(n)\right) \\
&+P\left(|v(n)|^{-N}[L(n)-L(n ; r|v(n)|)]>x \varepsilon \mid X(0) \in A(n)\right) .
\end{aligned}
$$

Applying Markov's inequality, the last member above is at most

$$
\left(|v(n)|^{-N} / x \varepsilon\right) \int_{\left\{t \in D^{N}:|t|>r|v(n)|\right\}} P(X(t) \in A(n) \mid X(0) \in A(n)) d t
$$

and so, by assumption,

$$
\lim _{r \rightarrow \infty} \liminf _{n \rightarrow \infty} b(x(1-\varepsilon) ; n ; r) \leq \liminf _{n \rightarrow \infty} a(x ; n) .
$$

Thus

$$
B(x-) \leq \liminf _{n \rightarrow \infty} a(x ; n) \leq \limsup _{n \rightarrow \infty} a(x ; n) \leq B(x+)
$$

and the lemma follows. 
THEOREM (3-3). Suppose that

(i) $v(n) \rightarrow 0$, as $n \rightarrow \infty$, and $=0$.

(ii) $\lim _{r \rightarrow \infty} \lim \sup _{n \rightarrow \infty}|v(n)|^{-N} \int_{\{|t|>r|v(n)|\}} P(X(t) \in A(n) \mid X(0) \in A(n)) d t$

Suppose, moreover, that on some probability space (we will, with an abuse of notation, also denote probabilities and expectations on this latter space by $P$ and $E)$ there exists a jointly measurable stochastic process $\left\{\eta(t): t \in K^{N}\right\}$ such that $\eta(t) \in\{0,1\}$ for all $t \in K^{N}$ and

(*) $E \eta\left(s_{1}\right) \cdots \eta\left(s_{m}\right)=\lim _{n \rightarrow \infty} P\left(X\left(v(n) s_{i}\right) \in A(n), i=1, \ldots, m \mid X(0) \in A(n)\right)$

for $m=1,2, \ldots$ and $s_{1}, \ldots, s_{m} \in K^{N}$. If for $x>0$

$$
G(x)=P\left(\int_{K^{N}} \eta(t) d t \leq x\right)
$$

then

$$
\lim _{n \rightarrow \infty} \frac{\int_{[0, z]} y d P\left(|v(n)|^{-N} L(n) \leq y\right)}{E\left(|v(n)|^{-N} L(n)\right)}=G(z)
$$

whenever $z$ is a continuity point of $G$.

Proof (see Theorem 7-1 of Berman (1985)). Define

$$
\xi(s ; n)= \begin{cases}I(X(v(n) s) \in A(n)), & |s| \leq|v(n)|^{-1}, \\ 0, & \text { otherwise }\end{cases}
$$

so that

$$
\lim _{n \rightarrow \infty} E\left(\xi\left(s_{1} ; n\right) \cdots \xi\left(s_{m} ; n\right) \mid X(0) \in A(n)\right)=E\left(\eta\left(s_{1}\right) \cdots \eta\left(s_{m}\right)\right)
$$

by assumption. Now if $r|v(n)| \leq 1$ we have that

$$
|v(n)|^{-N} L(n ; r|v(n)|)=\int_{\left\{t \in K^{N}:|t| \leq r\right\}} \xi(t ; n) d t
$$

and so for $m \in\{1,2, \ldots\}$

$$
\begin{aligned}
& E\left(\left(|v(n)|^{-N} L(n ; r|v(n)|)\right)^{m} \mid X(0) \in A(n)\right) \\
& \quad=\int_{\left|s_{1}\right| \leq r} \cdots \int_{\left|s_{m}\right| \leq r} E\left(\xi\left(s_{1} ; n\right) \cdots \xi\left(s_{m} ; n\right) \mid X(0) \in A(n)\right) d s_{1} \cdots d s_{m} \\
& \quad \rightarrow E\left[\left(\int_{|t| \leq r} \eta(t) d t\right)^{m}\right] \quad \text { as } n \rightarrow \infty .
\end{aligned}
$$

Since $|v(n)|^{-N} L(n ; r|v(n)|) \leq r^{N}$ we may apply the Moment Convergence Theorem to see that

$$
\lim _{n \rightarrow \infty} P\left(|v(n)|^{-N} L(n ; r|v(n)|) \leq x \mid X(0) \in A(n)\right)=P\left(\int_{|t| \leq r} \eta(t) d t \leq x\right)
$$

at all continuity points $x>0$ of the right-hand side. The theorem now follows upon letting $r \rightarrow \infty$ and then applying Lemma (3-2). 
COROLLARY (3-4). Let $X \in \mathcal{G}$ be a jointly measurable stationary process with covariance kernel given by $(s, t) \mapsto \gamma(s-t)$ for some function $\gamma: D^{N} \rightarrow \mathbf{R}$ with $\gamma(0)=1$. Assume that there exist sequences $\{v(n)\}_{n=1}^{\infty} \subset D \backslash\{0\}$ and $\left.\{u(n)\}_{n=1}^{\infty} \subset\right] 0, \infty\left[\right.$ and a continuous function $\psi: K^{N} \rightarrow[0, \infty[$, such that

(i) $v(n) \rightarrow 0$ as $n \rightarrow \infty$;

(i) $\psi \not \equiv 0$;

(iii) $\psi(t)=\lim _{n \rightarrow \infty} u(n)^{2}(1-\gamma(v(n) t)), t \in K^{N}$;

(iv) $\lim _{d \rightarrow \infty} \lim \sup _{n \rightarrow \infty} \int_{d \leq|s| \leq|v(n)|^{-1}} \exp \left(-u(n)^{2}(1-\gamma(v(n) s)) / 4\right) d s=0$. Then $(s, t) \mapsto \psi(s)+\psi(t)-\psi(s-t)$ is a continuous covariance kernel on $K^{N} \times$ $K^{N}$; and so we may construct on some probability space a centred, measurable Gaussian process $V$ with this covariance kernel and an independent exponential random variable $\tau$ with $E \tau=1$. If we set $A(n)=\mid u(n), \infty[$ then

$$
\begin{aligned}
& \lim _{n \rightarrow \infty} \frac{|v(n)|^{N} P\left(|v(n)|^{-N} L(n)>x\right)}{P(X(0)>u(n))} \\
& \quad=\int_{x}^{\infty} y^{-1} d P\left(\int_{K^{N}} I(V(t)-\psi(t)+\tau>0) d t \leq y\right)
\end{aligned}
$$

at each continuity point $x>0$ of the right-hand side.

Proof (see Theorem 8-1 in Berman (1985)).

Observe that $(s, t) \mapsto \gamma(s-t)-\gamma(s)-\gamma(t)+1, s, t \in D^{N}$, is the covariance kernel of the process $\left\{X(t)-X(0): t \in D^{N}\right\}$. Thus $\Gamma: K^{N} \times K^{N} \rightarrow \mathbf{R}$ given by

$$
\Gamma(s, t)= \begin{cases}\gamma(s-t)-\gamma(s)-\gamma(t)+1, & s, t \in D^{N}, \\ 0, & \text { otherwise, }\end{cases}
$$

is a covariance kernel. From condition (iii) we have

$$
\lim _{n \rightarrow \infty} u(n)^{2} \Gamma(v(n) s, v(n) t)=\psi(s)+\psi(t)-\psi(s-t)
$$

and so it is clear that the function on the right-hand side is a covariance kernel as claimed.

We now check conditions (i) and (ii) of Theorem (3-3). Condition (i) is obvious. As on page 16 of Berman (1982) we have

$$
P(X(s)>u \mid X(0)>u) \leq \kappa \exp \left(-u^{2}(1-\gamma(s)) / 4\right)
$$

for some constant $\kappa$ and so condition (ii) follows from condition (iv) above.

In order to be able to apply Theorem (3-3) it will suffice to show that the condition (*) holds with $\eta(t)=I(V(t)-\psi(t)+\tau>0)$. Let $\varphi$ and $\Phi$ denote, respectively, the standard normal density and distribution function. Then if $\sup _{1 \leq i \leq m}\left|s_{i}\right| \leq|v(n)|^{-1}$ we have

$$
\begin{aligned}
& P\left(X\left(v(n) s_{i}\right)>u(n), \forall i \mid X(0)>u(n)\right) \\
& =\int_{0}^{\infty} P\left(u(n)\left(X\left(v(n) s_{i}\right)-X(0)\right)+z>0, \forall i \mid X(0)=u(n)+z / u(n)\right) f(z ; n) d z
\end{aligned}
$$


where

$$
f(z ; n)=\frac{\varphi(u(n)+z / u(n))}{u(n)(1-\Phi(u(n)))}
$$

Now

$$
\begin{aligned}
\lim _{u \rightarrow \infty} \frac{\varphi(u+z / u)}{u(1-\Phi(u))} & =\lim _{u \rightarrow \infty} \frac{\varphi(u)}{u(1-\Phi(u))} \exp (-z) \exp \left(-z^{2} / 2 u^{2}\right) \\
& =\exp (-z)
\end{aligned}
$$

by a familiar approximation (see, for example, Lemma 2, Section VII.1 of Feller (1957)), so we need only show for each $z>0$ that if we set

$$
Y(t)= \begin{cases}X(t)-X(0), & t \in D^{N} \\ 0, & \text { otherwise, }\end{cases}
$$

then the finite dimensional distributions of $u(n) Y(v(n) \cdot)$ given $X(0)=u(n)+$ $z / u(n)$ converge to those $V(\cdot)-\psi(\cdot)$. Since the processes with which we are dealing are all Gaussian and $Y(0)=0$, it suffices to observe that

$$
\begin{aligned}
& E(u(n) Y(v(n) t) \mid X(0)=u(n)+z / u(n)) \\
& \quad=u(n)(\gamma(v(n) t)-1)(u(n)+z / u(n)) I\left(|t| \leq|v(n)|^{-1}\right) \\
& \quad \rightarrow-\psi(t) \quad \text { as } n \rightarrow \infty
\end{aligned}
$$

and when $|s|,|t| \leq|v(n)|^{-1}$

$$
\begin{aligned}
& \operatorname{Var}(X(v(n) s)-X(v(n) t) \mid X(0)=u(n)+z / u(n)) \\
& \quad=2[1-\gamma(v(n)(s-t))]\left[1-(\gamma(v(n) s)-\gamma(v(n) t))^{2}\right]
\end{aligned}
$$

so that

$$
\begin{gathered}
\operatorname{Var}(u(n) Y(v(n) s)-u(n) Y(v(n) t) \mid X(0)=u(n)+z / u(n)) \\
\rightarrow 2 \psi(s-t)=\operatorname{Var}(V(s)-V(t)) \quad \text { as } n \rightarrow \infty
\end{gathered}
$$

We may now apply Theorem (3-3) and thence Lemma (3-1) to complete the proof.

EXAMPLE. If $X(\cdot ; \alpha, \beta, k)$ is a member of the class of continuous processes described in the Example following Lemma (2-4) then it is easy to check that the conditions of Corollary (3-4) hold with $v(n)=\rho^{n}$ and $u(n)=q^{n \alpha / 2}$.

\section{A maximum limit theorem}

Our first aim in this section is to prove the following result. 
THEOREM (4-1). Let $X$ be a continuous process satisfying the conditions of Corollary (3-4). Suppose, moreover, that

$$
\psi(\cdot)=\lim _{n \rightarrow \infty} u(n)^{2}(1-\gamma(v(n) \cdot))
$$

uniformly on $D^{N}$, and that if we set

$$
\Psi(u)=\sup _{|t| \leq u} \sup _{n} u(n)^{2}(1-\gamma(v(n) t)), \quad u>0,
$$

then

$$
\int_{0}^{\varepsilon}(\Psi(u))^{1 / 2} u^{-1}\left(\log u^{-1}\right)^{-1 / 2} d u<\infty
$$

for some $\varepsilon>0$. Under these conditions

$$
\begin{aligned}
0 & <\lim _{n \rightarrow \infty} \frac{|v(n)|^{N} P\left(\max _{\left.t \in D^{N} X(t)>u(n)\right)}\right.}{P(X(0)>u(n))} \\
& =\int_{0+}^{\infty} y^{-1} d P\left(\int_{K^{N}} I(V(t)-\psi(t)+\tau>0) d t \leq y\right)<\infty .
\end{aligned}
$$

The proof of Theorem (4-1) will be via a sequence of lemmas and we use the techniques of Sections 13 and 14 in Berman (1982). Assume for the rest of this section that $X, V$ and $\tau$ are fixed as above.

LEMMA (4-2). Define

$$
Y_{n}(t)=u(n)(X(v(n) t)-X(0)), \quad t \in D^{N} .
$$

For fixed $z \in \mathbf{R}$ let $P_{n}$ denote the image measure on $C\left(D^{N}, \mathbf{R}\right)$ induced by the process $Y_{n}$ conditioned on $X(0)=u(n)+z / u(n)$. Then the sequence $\left\{P_{n}\right\}_{n=1}^{\infty}$ converges weakly to the image measure induced by the process

$$
\left\{V(t)-\psi(t): t \in D^{n}\right\} .
$$

ProOF. In the proof of Corollary (3-4) we have seen that the finite dimensional distributions of $P_{n}$ converge to those of $\left\{V(t)-\psi(t): t \in D^{N}\right\}$ so we need only show that the sequence $\left\{P_{n}\right\}$ is tight. Since $E\left(Y_{n}(t) \mid X(0)=u(n)+z / u(n)\right)$ converges uniformly to $-\psi(t)$ on $D^{N}$ as $n \rightarrow \infty$, it suffices to check the conditions of Corollary (2-6) for the sequence of processes defined by

$$
Z_{n}(t)=Y_{n}(t)-E\left(Y_{n}(t) \mid X(0)=u(n)+z / u(n)\right), \quad t \in D^{N},
$$

conditioned on $X(0)=u(n)+z / u(n)$. Now from the proof of Corollary (3-4) we have that

$$
\sup _{n} E\left(Z_{n}(t)^{2} \mid X(0)=u(n)+z / u(n)\right) \leq 2 \Psi(|t|)
$$

and it is clear that condition (i) of Corollary (2-6) holds. Similarly,

$$
\sup _{n} E\left(\left(Z_{n}(s)-Z_{n}(t)\right)^{2} \mid X(0)=u(n)+z / u(n)\right) \leq 2 \Psi(|s-t|)
$$


and hence that condition (ii) of Corollary (2-6) also holds.

NOTATION. Set

$$
M(n)=\max _{t \in D^{N}} X(v(n) t)
$$

and, in the notation of Section 3 ,

$$
\Lambda(n)=|v(n)|^{-N} L(n ;|v(n)|)
$$

for $A(n)=] u(n), \infty[$.

LEMMA (4-3). For almost all $z$

$$
\lim _{x \rightarrow 0} \limsup _{n \rightarrow \infty} P(M(n)>u(n), \Lambda(n) \leq x \mid X(0)=u(n)+z / u(n))=0 .
$$

Proof. Fix a continuous function $g:]-\infty, \infty[\rightarrow[0,1]$ such that $g(]-\infty, 0])=$ $\{0\}$ and $g(] 0, \infty[) \subset] 0,1]$. In the notation of Lemma (4-2) the conditional probability above is at most

$$
P\left(\max _{t \in D^{N}} Y_{n}(t)>-z, \int_{D^{N}} g\left(Y_{n}(t)+z\right) d t \leq x \mid X(0)=u(n)+z / u(n)\right) .
$$

Whenever $z$ is a point of continuity of the distribution of $\max _{t}(V(t)-\psi(t))$ it follows from Lemma (4-2) that the lim sup as $n \rightarrow \infty$ of the last expression is at most

$$
P\left(\max _{t \in D^{N}}(V(t)-\psi(t))>-z, \int_{D^{N}} g(V(t)-\psi(t)+z) d t \leq x\right) .
$$

The conclusion of the lemma is then clear from the continuity of $V$.

LEMma (4-4). There exists a function $R:[0, \infty[\rightarrow[0,1]$ such that

$$
R(x)=o\left(\exp \left(-c x^{2}\right)\right) \quad \text { as } \quad x \rightarrow \infty
$$

for some $c>0$ and for each $n$ and $u^{\prime} \leq u$ we have

$$
P\left(M(n)>u, X(0) \leq u^{\prime}\right) \leq \int_{-\infty}^{u^{\prime}} R(u(n)(u-y)) \varphi(y) d y .
$$

Proof. Since $|E(X(t) \mid X(0))| \leq|X(0)|$ we have that

$$
\begin{aligned}
P(M(n) & \left.>u, X(0) \leq u^{\prime}\right) \\
& \leq \int_{-\infty}^{u^{\prime}} P\left(\sup _{t \in D^{N}}|X(v(n) t)-E(X(v(n) t) \mid X(0)=y)|\right. \\
& >u-y \mid X(0)=y) \varphi(y) d y .
\end{aligned}
$$

By now familiar calculations observe that

$$
\operatorname{Var}(X(v(n) s)-X(v(n) t) \mid X(0)=y) \leq 2 u(n)^{-2} \Psi(|s-t|) .
$$


It is straightforward to check from the conditions of Theorem (4-1) that

$$
\sum_{r=1}^{\infty}\left(\log q^{2^{r}}\right)^{1 / 2}\left(\Psi\left(q^{-2^{r}}\right)\right)^{1 / 2}<\infty
$$

(see, for example, equation IV-1-14 in Jain and Marcus (1978)) and the lemma follows readily from Lemma (2-7).

LEMMA (4-5). We have

$$
\lim _{Q \rightarrow \infty} \limsup _{n \rightarrow \infty} \frac{P(X(0) \leq u(n)-Q / u(n), M(n)>u(n))}{1-\Phi(u(n))}=0 .
$$

PROOF. From Lemma (4-4) we see that the general term above is bounded by

$$
\begin{aligned}
\int_{-\infty}^{u(n)-Q / u(n)} & R(u(n)(u(n)-y)) \varphi(y) d y(1-\Phi(u(n)))^{-1} \\
& =u(n)^{-1} \int_{Q}^{\infty} R(z) \varphi(u(n)-z / u(n)) d z(1-\Phi(u(n)))^{-1} \\
& \leq \frac{\varphi(u(n))}{u(n)(1-\Phi(u(n)))} \int_{Q}^{\infty} R(z) \exp (z) d z
\end{aligned}
$$

and the result follows from the properties of $R$ and the approximation to the normal tail used in the proof of Corollary (3-4).

LEMMA (4-6). In the above notation

$$
\lim _{x \rightarrow 0} \limsup _{n \rightarrow \infty} \frac{P(M(n)>u(n), \Lambda(n) \leq x)}{1-\Phi(u(n))}=0 .
$$

PrOOF. From Lemma (4-5) it suffices to show for arbitrary $Q>0$ that

$$
\lim _{x \rightarrow 0} \limsup _{n \rightarrow \infty} \frac{P(M(n)>u(n), \Lambda(n) \leq x, X(0)>u(n)-Q / u(n))}{1-\Phi(u(n))}=0 .
$$

The expression over which we are taking limits is at most

$$
\begin{gathered}
\frac{\varphi(u(n))}{u(n)(1-\Phi(u(n)))} \int_{-Q}^{\infty} P(M(n)>u(n), \\
\Lambda(n) \leq x \mid X(0)=u(n)+y / u(n)) \exp (-y) d y
\end{gathered}
$$

and the lemma now follows from Lemma (4-3).

PROOF OF THEOREM (4-1). Set

$$
J=\int_{0+}^{\infty} y^{-1} d P\left(\int_{K^{N}} I(V(t)-\psi(t)+\tau>0) d t \leq y\right) .
$$


It is clear from Corollary (3-4) that

$$
\liminf _{n \rightarrow \infty} \frac{|v(n)|^{N} P\left(\max _{t \in D^{N}} X(t)>u(n)\right)}{P(X(0)>u(n))} \geq J .
$$

Conversely, observe that for any $x>0$

$$
\begin{aligned}
& \frac{|v(n)|^{N} P\left(\max _{t \in D^{N}} X(t)>u(n)\right)}{P(X(0)>u(n))} \\
& \leq|v(n)|^{N} P\left(|v(n)|^{-N} L(n)>x\right)(1-\Phi(u(n)))^{-1} \\
& \quad+|v(n)|^{N} P\left(\max _{t \in D^{N}} X(t)>u(n),|v(n)|^{-N} L(n) \leq x\right)(1-\Phi(u(n)))^{-1} .
\end{aligned}
$$

By Corollary (3-4) the first term on the right-hand side converges to $J$ as $n \rightarrow \infty$ and then $x \downarrow 0$. Splitting $D^{N}$ into $|v(n)|^{-N}$ balls of diameter $|v(n)|$ we see that the second term is bounded by

$$
P(M(n)>u(n), \Lambda(n) \leq x)(1-\Phi(u(n)))^{-1},
$$

which converges to 0 as $n \rightarrow \infty$ and then $x \downarrow 0$ by Lemma (4-6).

It only remains to check that $0<J<\infty$. In order to show that $J>0$ we need to prove that

$$
P\left(\int_{K^{N}} I(V(t)-\psi(t)+\tau>0) d t<\infty\right)>0
$$

Since

$$
\begin{aligned}
P\left(\int_{K^{N}} I\right. & (V(t)-\psi(t)+\tau>0) d t<\infty) \\
& \geq P\left(\int_{K^{N}} I(V(t)-\psi(t)+1>0) d t<\infty ; \tau \leq 1\right) \\
& \geq P\left(\int_{K^{N}} I(V(t)-\psi(t)+1>0) d t<\infty\right) P(\tau \leq 1)
\end{aligned}
$$

and $P(\tau \leq 1)>0$ it will certainly suffice to show that

$$
\int_{K^{N}} P(V(t)>\psi(t)-1) d t<\infty
$$

This last integral is bounded by

$$
\begin{aligned}
\int_{K^{N}} & \exp \left(-(\psi(t)-1)^{2} / 4 \psi(t)\right) d t \\
& \leq e^{1 / 2} \int_{K^{N}} \exp (-\psi(t) / 4) d t \\
& =e^{1 / 2} \int_{K^{N}} \lim _{n \rightarrow \infty} \exp \left(-u(n)^{2}(1-\gamma(v(n) t)) / 4\right) I\left(|t| \leq|v(n)|^{-1}\right) d t \\
& \leq e^{1 / 2} \limsup _{n \rightarrow \infty} \int_{|t| \leq|v(n)|^{-1}} \exp \left(-u(n)^{2}(1-\gamma(v(n) t)) / 4\right) d t \\
& <\infty,
\end{aligned}
$$


where the last inequality follows from condition (iv) of Corollary (3-4).

Finally, we show that $J<\infty$. Now

$$
\begin{aligned}
& P\left(\max _{t \in D^{N}} X(t)>u(n)\right) \leq|v(n)|^{-N} P(M(n)>u(n)) \\
& \quad \leq|v(n)|^{-N}(P(X(0)>u(n))+P(M(n)>u(n), X(0) \leq u(n)))
\end{aligned}
$$

and so, by Lemma (4-4),

$$
\begin{aligned}
\frac{|v(n)|^{N} P\left(\max _{t \in D^{N}} X(t)>u(n)\right)}{1-\Phi(u(n))} & \\
& \leq 1+\int_{-\infty}^{u(n)} R(u(n)(u(n)-y)) \frac{\varphi(y)}{(1-\Phi(u(n)))} d y \\
& \leq 1+\frac{\varphi(u(n))}{u(n)(1-\Phi(u(n)))} \int_{0}^{\infty} R(z) \exp (z) d z .
\end{aligned}
$$

From the properties of $R$ this last integral is seen to be bounded.

EXAMPLE. It is straightforward to check that the processes given in the Example following Corollary (3-4) satisfy the extra conditions of Theorem (4-1).

By applying Slepian's Lemma to the conclusions of the previous Example we may obtain some information about the maximum of a process which is no longer assumed to be stationary.

COROLlaRY (4-7). Suppose that $X \in \mathcal{G}$ is a continuous process for which

$$
E X(t)^{2}=1, \quad t \in D^{N},
$$

and

$$
\begin{aligned}
0 & <\lim _{u \downarrow 0} \inf _{|s-t| \leq u} \frac{E(X(s)-X(t))^{2}}{|s-t|^{\alpha}} \\
& \leq \lim _{u \downarrow 0} \sup _{|s-t| \leq u} \frac{E(X(s)-X(t))^{2}}{|s-t|^{\alpha}}<\infty .
\end{aligned}
$$

Then

$$
\begin{aligned}
0 & <\liminf _{n \rightarrow \infty} \frac{P\left(\max _{t \in D^{N}} X(t)>q^{n \alpha / 2}\right)}{q^{n N} P\left(X(0)>q^{n \alpha / 2}\right)} \\
& \leq \limsup _{n \rightarrow \infty} \frac{P\left(\max _{t \in D^{N}} X(t)>q^{n \alpha / 2}\right)}{q^{n N} P\left(X(0)>q^{n \alpha / 2}\right)}<\infty .
\end{aligned}
$$

Proof. We may find $\beta^{\prime}>0$ and $j \in \mathbf{N}$ such that $E(X(s)-X(t))^{2} \leq \beta^{\prime}|s-t|^{\alpha}$ whenever $|s-t| \leq q^{-j}$. Choose $k^{\prime}>j$ such that $q^{k^{\prime} \alpha} \geq \beta^{\prime}$. Consider the process 
$X\left(\cdot ; \alpha, \beta^{\prime}, k^{\prime}\right)$ and note that

$$
E X\left(s ; \alpha, \beta^{\prime}, k^{\prime}\right) X\left(t ; \alpha, \beta^{\prime}, k^{\prime}\right) \leq E X(s) X(t), \quad s, t \in D^{N}
$$

From Slepian's Lemma (see, for example, Lemma II-4-3 of Jain and Marcus (1978)) we have

$$
P\left(\max _{t \in D^{N}} X(t)>x\right) \leq P\left(\max _{t \in D^{N}} X\left(t ; \alpha, \beta^{\prime}, k^{\prime}\right)>x\right)
$$

for all $x \in \mathbf{R}$.

Conversely, we may find $\left.\left.\beta^{\prime \prime} \in\right] 0,1\right]$ and $k^{\prime \prime} \in \mathbf{N}$ such that $E(X(s)-X(t))^{2} \geq$ $2 \beta^{\prime \prime}|s-t|^{\alpha}$ whenever $|s-t| \leq q^{-k^{\prime \prime}}$. Again applying Slepian's Lemma we have

$$
\begin{aligned}
P\left(\max _{t \in D^{N}} X(t)>x\right) & \geq P\left(\max _{t \in\left(B^{k^{\prime \prime}}\right)^{N}} X(t)>x\right) \\
& \geq P\left(\max _{t \in\left(B^{\prime \prime}\right)^{N}} X\left(t ; \alpha, \beta^{\prime \prime}, 0\right)>x\right) \\
& \geq P\left(\max _{t \in D^{N}} X\left(t ; \alpha, \beta^{\prime \prime} q^{-k^{\prime \prime} \alpha}, 0\right)>x\right)
\end{aligned}
$$

for all $x \in \mathbf{R}$.

The corollary now follows from the conclusions of the previous example.

\section{References}

Yu. K. Belyaev and V. I. Piterbarg (1972), 'Asymptotics of the average number of A-points of overshoot of a Gaussian field beyond a high level', Dokl. Akad. Nauk SSSR 203, 309-313.

S. M. Berman (1982), 'Sojourns and extremes of stationary processes', Ann. Probab. 10, 1-46.

S. M. Berman (1985), 'Limit theorems for sojourns of stochastic processes', Probability in Banach spaces V, Proceedings, Medford 1984 (Lecture Notes in Mathematics 1153, SpringerVerlag, Berlin).

P. Billingsley (1968), Convergence of probability measures (John Wiley and Sons, New York).

S. N. Evans (1986), 'Sample path properties of Gaussian stochastic processes indexed by a local field', Proc. London Math. Soc., to appear.

S. N. Evans (1988), 'Continuity properties of Gaussian stochastic processes indexed by a local field', Proc. London Math. Soc. (3) 56, 380-416.

W. Feller (1957), An introduction to probability theory and its applications, Volume I (Second Edition) (John Wiley and Sons, New York).

N. C. Jain and M. B. Marcus (1978), 'Continuity of sub-Gaussian processes', Advances in Probability, Volume 4 (M. Dekker, New York). 
J. Pickands (1969a), 'Upcrossing probabilities for stationary Gaussian processes', Trans. Amer. Math. Soc. 145, 51-73.

J. Pickands (1969b), 'Asymptotic properties of the maximum in a stationary Gaussian process', Trans. Amer. Math. Soc. 145, 75-86.

M. H. Taibleson (1975), Fourier analysis on local fields (Princeton University Press, Princeton, and University of Tokyo Press, Tokyo.)

Department of Mathematics

University of Virginia

Mathematics-Astronomy Building

Charlottesville, Virginia 22903

U.S.A. 